\title{
What is Transition Temperature for Bitumen and How to Measure It?
}

\author{
M. R. Nivitha ${ }^{1}$ J. Murali Krishnan ${ }^{1}$ ()
}

Received: 17 September 2015/Accepted: 24 December 2015/Published online: 2 February 2016

(C) Springer International Publishing Switzerland 2016

\begin{abstract}
In a typical year and for any typical location in India, the lowest temperature of the pavement can go up to $0{ }^{\circ} \mathrm{C}$ or below and the highest temperature can reach up to $70{ }^{\circ} \mathrm{C}$ or above. During such temperature changes, the binder exhibits behaviour ranging from a glassy material (at lower temperature) to a viscoelastic/non-Newtonian fluid (at higher temperature). The range of temperature change which is of interest to a pavement engineer is the transition between viscoelastic solid to viscoelastic fluid. Two measurements are normally used for quantifying the transition temperature and they are the $G^{\prime}-G^{\prime \prime}$ cross over point and the point when $\tan \delta$ is independent of frequency (Winter-Chambon criterion). These measures are applicable when the material has a single relaxation time (typically a single constituent material). Since bitumen is a mixture of several complex hydrocarbons each with different relaxation time, it is natural that such measures are also frequency dependent. This investigation will focus attention on determining the frequency dependence of $\mathrm{G}^{\prime}-$ $\mathrm{G}^{\prime \prime}$ cross over point as well as the relationship of $\tan \delta$ with frequency. An unmodified binder of VG30 grade as per IS73-2013 and a crumb rubber modified binder of meeting CRMB 60 grade as per IS:15462-2004 were used in this investigation. To estimate the viscoelastic solid-fluid transition temperature, frequency domain tests were
\end{abstract}

3rd Conference of Transportation Research Group of India (3rd CTRG).

\author{
J. Murali Krishnan \\ jmk@iitm.ac.in \\ M. R. Nivitha \\ nivithamr@gmail.com \\ 1 Indian Institute of Technology Madras, Chennai, India
}

performed in the temperature range of $25-75{ }^{\circ} \mathrm{C}$ for frequencies in the range of 50-1 Hz. All the tests discussed in this study were performed at two aging conditions, unaged and short-term aged. Due to the frequency dependent nature of bitumen, the transition temperature is not a sharp point as in the case of polymers but occurred gradually over a temperature range. Contrary to what one expected, modification process did not drastically change the transition temperature and one could see a change of only $3{ }^{\circ} \mathrm{C}$ for CRMB 60 when compared to VG30. Aging of the modified bitumen proportionally shifted the transition temperature in a similar manner to that of the unmodified bitumen. It was also clearly seen that the Winter-Chambon criterion was not met for all the binders investigated in this study.

Keywords Transition $\cdot$ CRMB $\cdot$ Aging $\cdot$ Frequency
sweep

\section{Introduction}

The magnitude of pavement temperature plays a critical role in determining the behaviour of the pavement in a bituminous pavement. The properties of binder highly influence the rutting and fatigue characteristics of the pavement and hence the mechanical behaviour of the binder is the focus of this study. The pavement temperature in India varies from -10 to $70{ }^{\circ} \mathrm{C}$ depending on the location and the time period of the year. In such temperature ranges, the binder exhibits behaviour ranging from a glassy material (at lower temperature) to a viscoelastic/ non-Newtonian fluid (at higher temperature). Since the stress-strain response of the bituminous pavement depends to a large extent on the rheological behaviour of bitumen, it 
is required to understand the properties of bitumen in the service temperature range.

While it is easier to classify the response of the material at the higher (non-Newtonian fluid) and lower (glassy material) temperature, it is extremely complex to classify the response of the material when it is viscoelastic in nature since it could behave like a viscoelastic solid or a viscoelastic fluid. In fact, the rheological behaviour of bitumen keeps cycling between viscoelastic solid to viscoelastic fluid during most part of the year excepting of course for few hours in few days in which extreme thermomechanical behaviour is seen. Since the response of the pavement varies drastically when the binder behaves as a viscoelastic solid or a viscoelastic fluid, the quantification of such response is necessary. It is also necessary to find out the temperature and/or time period during which such gradual cross over across viscoelastic solid-fluid behaviour is seen.

In polymer literature, the gel point is defined as the temperature/frequency at which a solid-like material is formed from a liquid-like material [1], typically termed as gelation. At the gel point, the average molecular weight is considered to tend to infinity. The process of gelation can be physical or chemical in nature. In chemical gels, chemically cross-linked networks are formed at the gel point which is irreversible while in physical gels, physical cross links are observed between molecules which are reversible in nature. For bitumen, the viscoelastic transition from a fluid-like behaviour to a solid-like behaviour as the temperature is reduced is comparable to that of the gelation occurring in polymers. It is more appropriate to associate this transition in bitumen to physical gelation as the viscoelastic solidfluid transition is reversible in nature by varying the temperature or frequency. The (physical) gel point for polymers can thus be termed as viscoelastic solid-fluid transition temperature for bitumen.

This transition temperature was observed as a sharp point for polymers as a single constituent/mechanism is responsible for transition. However, bitumen is a multi constituent material with different constituents having different properties. For instance, if the Corbett fractions are considered for bitumen, saturates are made of linear long chain aliphatics while asphaltenes are made up of aromatic structures. The gelation characteristics of each constituent are different and can occur at different combinations of temperature and frequencies. Due to this reason, the transition temperature for bitumen is not a sharp point but a regime in the temperature-frequency domain. This has been shown by Padmarekha and Krishnan [2] where the transition temperature has been observed to vary from 20 to $50{ }^{\circ} \mathrm{C}$ depending on the processing condition adopted for the production of bitumen.
Pavement engineers throughout the world use dynamic shear rheometer as the equipment of choice for testing unmodified and modified binders at different aging conditions. Using such equipment, the transition temperature can be characterized by carrying out a stress relaxation or creep and recovery test and by quantifying the residual stress and strain after sufficiently longer periods during unloading. While empirical measures such as Deborah number (ratio of time of relaxation to time of observation) can give guidelines on the choice of unloading time, it is possible that one may have to conduct experiments for sufficiently longer time, few hours to few days (loading/unloading) to quantify the same. However, the testing mode for bitumen always has been in frequency domain. A parallel plate geometry is normally used and the material is subjected to a chosen frequency and amplitude in a strain-controlled mode. The resulting torque is used to calculate the storage modulus $\left(\mathrm{G}^{\prime}\right)$, loss modulus $\left(\mathrm{G}^{\prime \prime}\right)$ and phase lag $(\tan \delta)$. Since these parameters depend on the frequency of testing, most of the specification compliance testing clearly spell out the frequency to be used during testing.

It will be interesting to see how the response of the material can be quantified as viscoelastic fluid or viscoelastic solid when subjecting them to frequency domain testing in small amplitude oscillatory shear. Two measurements are normally used for quantifying the transition temperature and they are the $\mathrm{G}^{\prime}-\mathrm{G}^{\prime \prime}$ cross over point and the point when $\tan \delta$ is independent of frequency (WinterChambon criterion). The storage modulus and loss modulus are calculated for oscillatory tests performed in the linear regime. The storage modulus indicates the magnitude of elastic component and loss modulus, the viscous component. Chambon and Winter [3] have shown that the $\mathrm{G}^{\prime}-\mathrm{G}^{\prime \prime}$ cross over can be used to identify the sol-gel transition in polymers. At the transition point, they have shown that,

$\mathrm{G}^{\prime}(\omega)=\mathrm{G}^{\prime \prime}(\omega)=\omega^{n}$.

It was later shown that the $\mathrm{G}^{\prime}-\mathrm{G}^{\prime \prime}$ cross over was valid only for specific type of polymers with $n=1 / 2$. For materials with other values of $n$, different methods of estimation of transition temperature was suggested [4].

One such parameter used to estimate the transition temperature is the frequency independence of the loss tangent, $\tan \delta$. Tan $\delta$ is flat at the point of gelation while at other temperatures, it decays with frequency. Hence this concept also can be used to identify the transition temperature from an oscillatory shear test. At the transition point, it was shown that $\mathrm{G}^{\prime}$ and $\mathrm{G}^{\prime \prime}$ have similar variation with frequency and hence $\mathrm{G}^{\prime \prime} / \mathrm{G}^{\prime}=\tan \delta=\tan (n \pi / 2)$ is independent of the frequency of testing. Attempts have been made to use frequency independent $\tan \delta$ for bitumen to identify the transition temperature [2]. However, there 
was no frequency independent zone observed for the unmodified bitumen. Nevertheless, this is used as a parameter to identify the transition temperature as frequency independent $\tan \delta$ has been observed for modified bitumen such as polyphosphoric acid modified bitumen [5].

The influence of two parameters, modifier and aging on the transition temperature is the focus of this study. Crumb rubber is chosen as the modifier as scarcely any study exists that focuses on estimating the transition temperature for crumb rubber modified bitumen. Crumb rubber possesses interesting characterises as a bitumen modifier in terms of the interaction with bitumen. The four major constituents of crumb rubber are styrene butadiene rubber, carbon black, metals and textile with zinc oxide and sulphur present in smaller proportion $(<1 \%)$ [6]. The interaction of crumb rubber with bitumen has been observed to be partly physical and partly chemical [7]. The physical interaction can be attributed to the rubber fraction while the constituents showing chemical interaction could not be delineated. It is now required to see the how the different constituents of modifier influence the transition temperature of the parent bitumen.

The influence of aging condition on the transition temperature is required to be studied as the presence of modifier has a significant influence on the aging characteristics of the base bitumen. For the base bitumen, short-term aging has been observed to shift the transition regime to higher temperatures [2]. However, no such understanding exists for the modified bitumen. The amount of carbonyl and sulfoxide formation, the compounds primarily responsible for increase in stiffness upon short-term aging are higher in an unmodified bitumen compared to crumb rubber modified bitumen [7]. This is attributed to the hindrance of the capability oxygen of molecules to oxidise the bitumen molecules due to the swollen network of the rubber molecules. Hence a modified bitumen is expected to show lesser increase in stiffness upon short-term aging compared to an unmodified bitumen. However, whether such a reduction in increase in stiffness influences the transition temperature needs to be assessed.

To summarize, estimation of the viscoelastic solid-fluid transition temperature for unmodified and modified bitumen is the focus of this study. Two types of bitumen, an unmodified and a crumb rubber modified are chosen to investigate the transition temperature. An oscillatory shear test is performed in the linear regime and parameters such as $G^{\prime}-G^{\prime \prime}$ cross over and frequency independence of $\tan \delta$ are chosen to estimate the transition temperature. The influence of modification on the transition regime was studied for crumb rubber since it exhibits both physical and chemical interaction with bitumen. The effect of short-term aging on the transition regime for both the binders was also assessed.

\section{Experimental Investigation}

\section{Materials}

Two materials were used in this study: a crumb rubber modified bitumen and the base bitumen used to manufacture the modified bitumen. The unmodified bitumen complied with VG30 grade as per IS 73:2013 [8] and the crumb rubber modified bitumen complied with CRMB 60 as per IS 15462:2004 [9]. The materials were tested in two aging conditions, unaged and short-term aged. The short-term aging was performed using a Rolling Thin Film Oven (RTFO) test as per ASTM D 2872:2012 [10].

\section{Test Procedure}

The rheological properties of unmodified and crumb rubber modified bitumen were measured using a dynamic shear rheometer. MCR-301 from Anton Paar was used to test the bitumen samples. A parallel plate geometry of $25 \mathrm{~mm}$ diameter was used for the entire temperature regime. A small amplitude oscillatory shear test was performed on bitumen in the linear regime.

The linear regime was estimated by performing an amplitude sweep test from $0.001 \%$ strain to $0.3 \%$ strain at the rate of $0.001 \%$ strain per second. The strain amplitude for which the modulus reduced to $95 \%$ of the initial strain was taken as the linear limit. The test was performed at the lowest test temperature $\left(25{ }^{\circ} \mathrm{C}\right.$ for unaged materials and $30{ }^{\circ} \mathrm{C}$ for short-term aged materials) so that the linear limit obtained at all other higher temperatures will be greater than that obtained for the lowest test temperature. The variation of modulus with amplitude for CRMB in shortterm aged condition is shown in Fig. 1. The modulus reduced to $95 \%$ of its initial value, $\mathrm{G}_{0}$ for strain amplitude

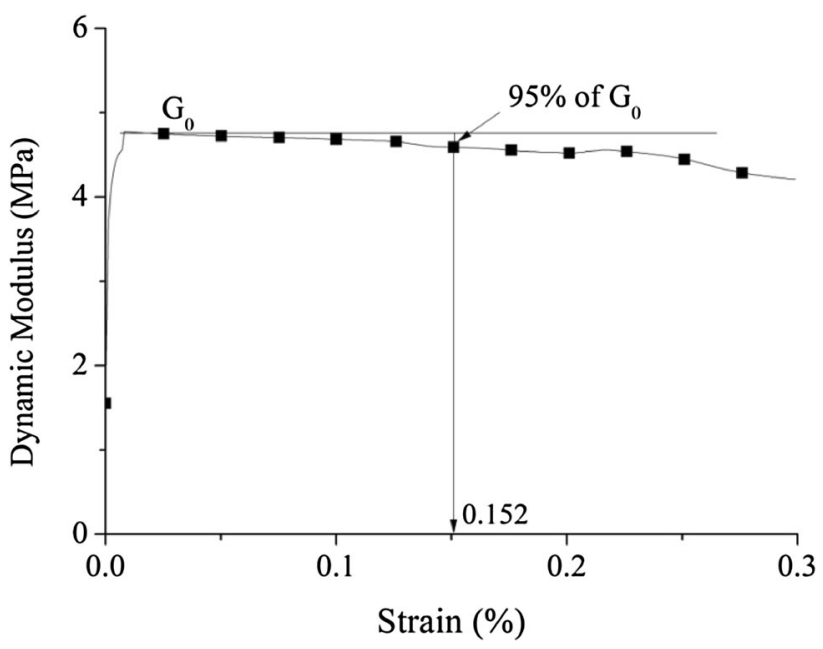

Fig. 1 Linear limit for CRMB in short-term aged condition 
of $0.152 \%$. Hence the linear limit for CRMB in short-term aged condition is taken as $0.152 \%$. Similarly, the linear limit was calculated for both materials in the two aging conditions. Based on this test, the linear limit was fixed as the lowest value obtained from all the four samples (two samples in two aging conditions). A strain amplitude of $0.07 \%$ was used for the frequency sweep test.

The frequency sweep test was performed on the samples by varying the frequency from 50 to $1 \mathrm{~Hz}$ at the rate of $0.1 \mathrm{~Hz}$ per second. The test frequency was limited to $50 \mathrm{~Hz}$ as beyond this frequency, the time of loading is expected to be very small such that it may not significantly influence the response of bitumen. The test was performed at different temperatures in the interval of $22-75^{\circ} \mathrm{C}$. A test temperature interval of $3{ }^{\circ} \mathrm{C}$ was chosen initially and it was increased to 5 and $10{ }^{\circ} \mathrm{C}$ beyond $40{ }^{\circ} \mathrm{C}$. The precise value of the temperature used for testing are mentioned at the appropriate places in the results and discussion section. All the experiments were performed twice to ensure repeatability. A $10 \%$ variation in modulus was fixed as the repeatability limit for all bitumen samples.

\section{Results and Discussion}

The variation of modulus with frequency in unaged condition is shown in Fig. 2a for the unmodified bitumen and crumb rubber modified bitumen at $25{ }^{\circ} \mathrm{C}$. It can be seen that at high frequencies $(20-50 \mathrm{~Hz})$, the crumb rubber modified bitumen shows lesser stiffness compared to unmodified bitumen. For shorter times of loading, the crumb rubber modified bitumen is observed to be 'softer' compared to the unmodified bitumen. However, for the same temperature at low frequencies (shown in the inset figure), one can observe that the crumb rubber modified bitumen shows higher stiffness compared to the unmodified bitumen. At $75{ }^{\circ} \mathrm{C}$, one can observe a similar increased stiffness for CRMB compared to VG30 for all frequencies (Fig. 2b).

This behaviour of the crumb rubber modified bitumen is desirable from the pavement performance perspective. At low temperatures $\left(<30{ }^{\circ} \mathrm{C}\right)$, the material is more vulnerable to fatigue cracking. Hence a reduced stiffness is desirable at low temperatures to limit the effect of fatigue cracking. Similarly at high temperatures, rutting is the predominant mode of failure. The increased stiffness of CRMB at higher temperatures will also reduce the effect of rutting in pavements.

In the short-term aged condition at $30{ }^{\circ} \mathrm{C}$, it can be seen that VG30 exhibits higher stiffness compared to CRMB at all frequencies as seen from Fig. 3a. This is similar to the behaviour observed in the unaged condition. At $75^{\circ} \mathrm{C}$, the increased stiffness of CRMB compared to VG30 is clearly seen for all materials (Fig. 3b). However the ratio of the stiffness of VG30 to CRMB is 0.385 for unaged condition while it is 0.608 in the short-term aged condition at $50 \mathrm{~Hz}$. The stiffness of CRMB has increased from 55,300 to 63,600 Pa (1.15 times) upon short-term aging. However, for the unmodified bitumen, the increase in stiffness is from 21,300 to $38,700 \mathrm{~Pa}$ which is a 1.81 times increase in modulus. This is in line with the observation made by Nivitha et al. [7]. They have shown that the formation of oxidation products responsible for increase in stiffness is much higher for the unmodified bitumen upon short-term aging compared to crumb rubber modified bitumen.

It is now necessary to check how the presence of modifier showing different stiffness characteristics at different temperatures compared to the unmodified bitumen will influence the transition temperature. Also the influence of reduced increase in stiffness on the transition regime for the crumb rubber modified bitumen upon short-term aging compared to the unmodified bitumen has to be assessed.

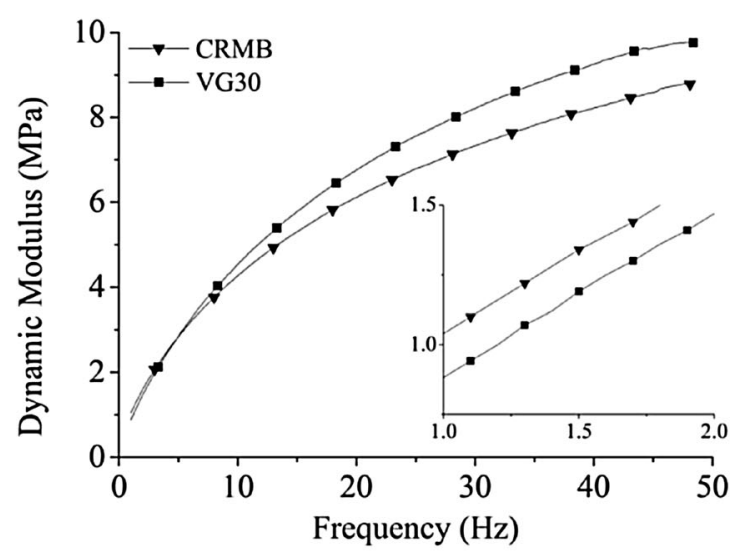

(a) $25^{\circ} \mathrm{C}$

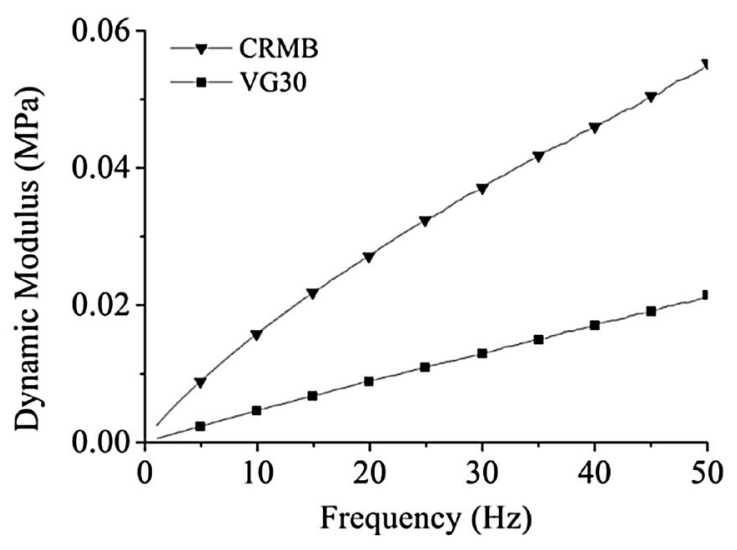

(b) $75^{\circ} \mathrm{C}$

Fig. 2 Variation of modulus with frequency in unaged condition 


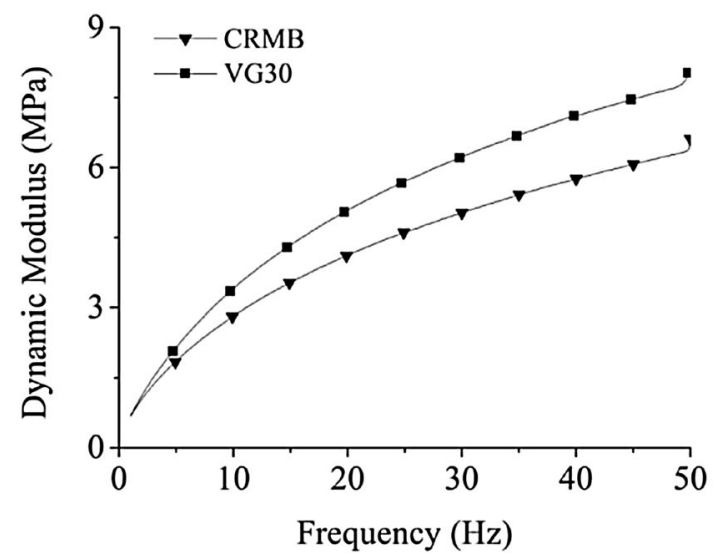

(a) $30^{\circ} \mathrm{C}$

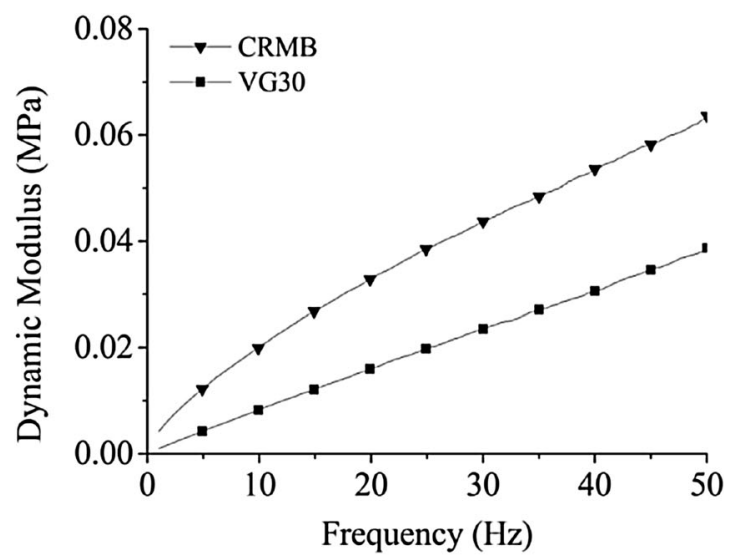

(b) $75^{\circ} \mathrm{C}$

Fig. 3 Variation of modulus with frequency in short-term aged condition

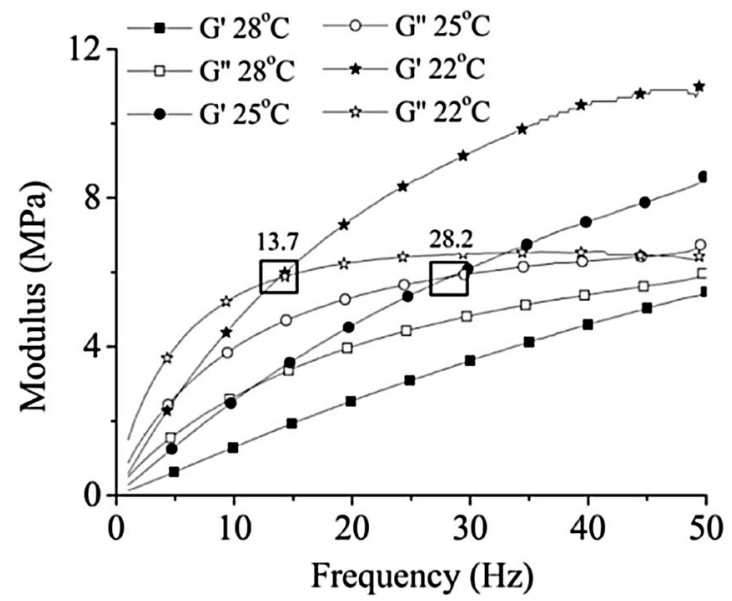

(a) VG30

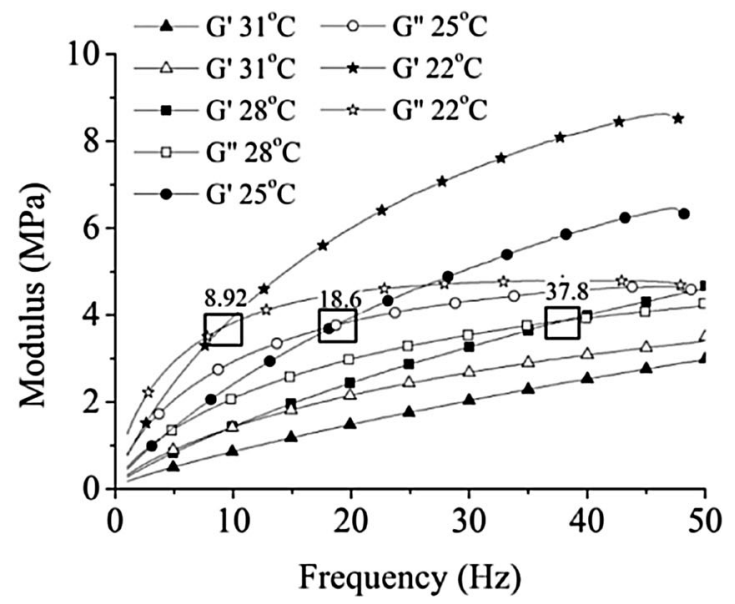

(b) CRMB

Fig. $4 G^{\prime}, G^{\prime \prime}$ cross over for unaged binders

\section{$\mathbf{G}^{\prime}, \mathbf{G}^{\prime \prime}$ Cross over}

The storage $\left(\mathrm{G}^{\prime}\right)$ and loss modulus $\left(\mathrm{G}^{\prime \prime}\right)$ variation with temperature is used to estimate the transition temperature for unmodified and modified bitumen. At low temperatures and high frequencies, say for instance $50 \mathrm{~Hz}$ at $22{ }^{\circ} \mathrm{C}$, the storage modulus is higher than loss modulus. As the frequency reduces, both storage and loss modulus reduce with temperature. However, the rate of reduction of storage modulus is much higher compared to loss modulus. Hence beyond a specified frequency, the loss modulus becomes higher than the storage modulus. The frequency at which such cross over between storage and loss modulus occurs (or in other words, the frequency at which both storage and loss modulus are equal) is taken as the transition temperature.

For the unmodified bitumen, the cross over is seen to occur at $13.7 \mathrm{~Hz}$ for $22{ }^{\circ} \mathrm{C}$ in the unaged condition
(Fig. 4a). This cross over shifted to $28.2 \mathrm{~Hz}$ at $25{ }^{\circ} \mathrm{C}$ and at $28^{\circ} \mathrm{C}$, the loss modulus is higher than the storage modulus for all frequencies. The temperature at which the cross over frequency just crosses $50 \mathrm{~Hz}$ can be expected between 25 and $28{ }^{\circ} \mathrm{C}$. The transition temperature for $\mathrm{VG} 30$ in unaged condition is taken as $25-28{ }^{\circ} \mathrm{C}$. As the temperature increases, one can see that the stiffness reduces appropriately and cross over frequency shifts to higher frequencies. For the crumb rubber modified bitumen, cross over frequency at $22{ }^{\circ} \mathrm{C}$ is observed at $8.92 \mathrm{~Hz}$ (Fig. 4b). Though CRMB exhibits less stiff behaviour compared to the unmodified bitumen at $22{ }^{\circ} \mathrm{C}$, the shift of cross over frequency to lower frequencies clearly illustrates the effect of modification, CRMB with the cross over frequency shifted to the left. The transition temperature obtained for CRMB in unaged condition is $28-31{ }^{\circ} \mathrm{C}$. Approximately, a $3{ }^{\circ} \mathrm{C}$ 


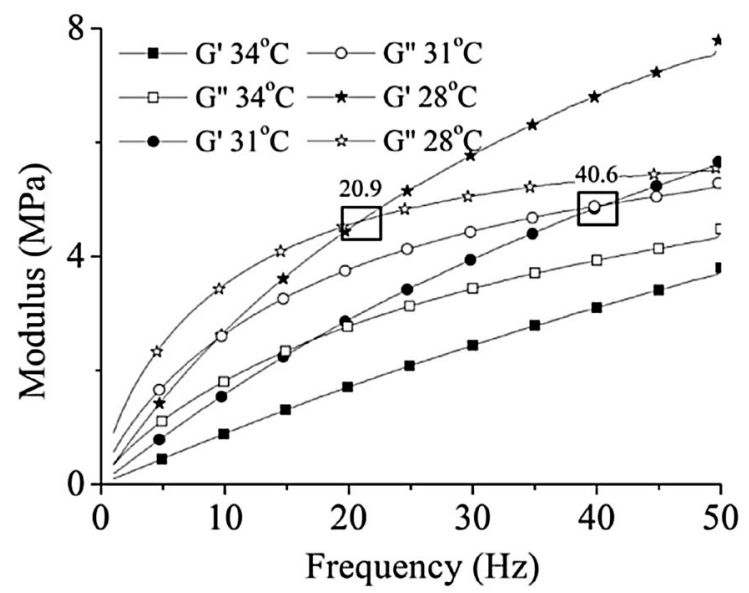

(a) VG30

Fig. $5 \mathrm{G}^{\prime}, \mathrm{G}^{\prime \prime}$ cross over for short-term aged binders

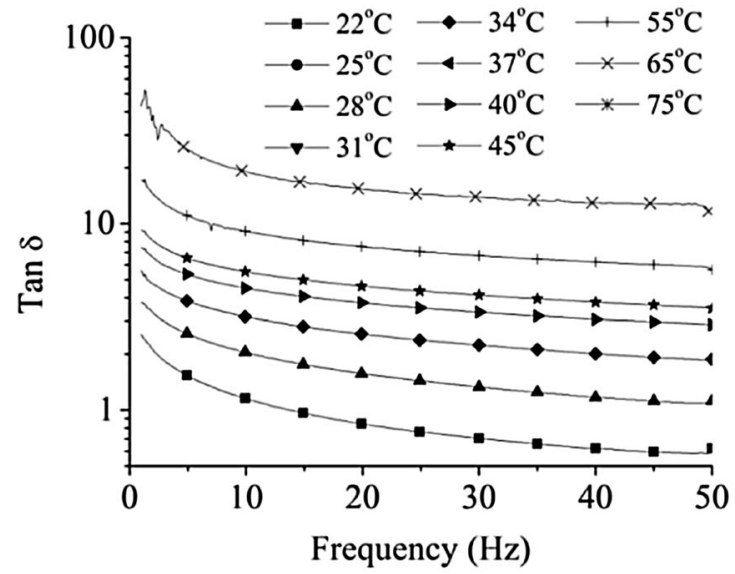

(a) VG30

Fig. 6 Tan $\delta$ in unaged condition

shift is seen for the modified bitumen compared to VG30 due to the effect of modification.

The cross over frequency shifted to lower frequencies upon short-term aging for both VG30 and CRMB (Fig. 5). Upon short-term aging, an increase in modulus is observed which shifts the cross over frequency to lower frequencies. It is to be noted here that the increase/reduction in stiffness due to temperature and aging have inverse relation with cross over frequency while the effect of modification showed otherwise. Thus the effect of aging and the effect of modification are considered to have different effects on the transition temperature. The transition temperature obtained for VG30 is $31-34{ }^{\circ} \mathrm{C}$ while that for CRMB is observed as $34-37{ }^{\circ} \mathrm{C}$ in the short-term aged condition. At $34{ }^{\circ} \mathrm{C}$, the storage modulus is equal to the loss modulus within the error percentage of $10 \%$ at least at $50 \mathrm{~Hz}$ frequency (shown with error bars plotted for storage modulus

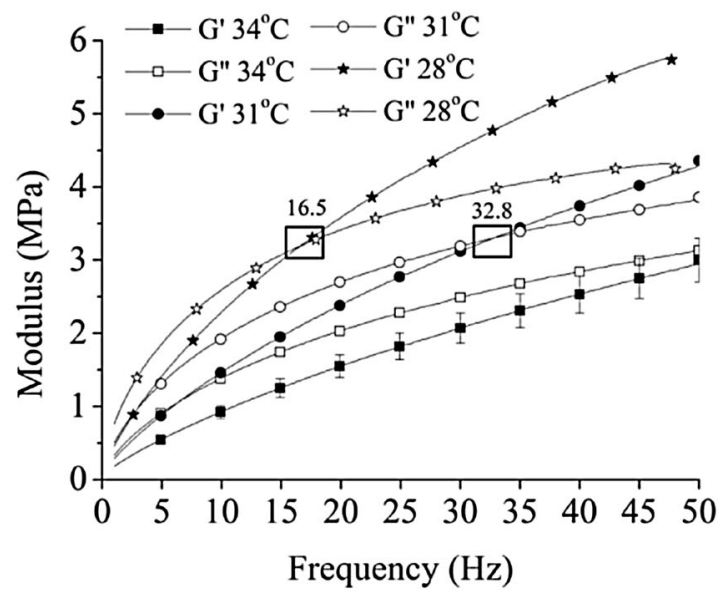

(b) CRMB

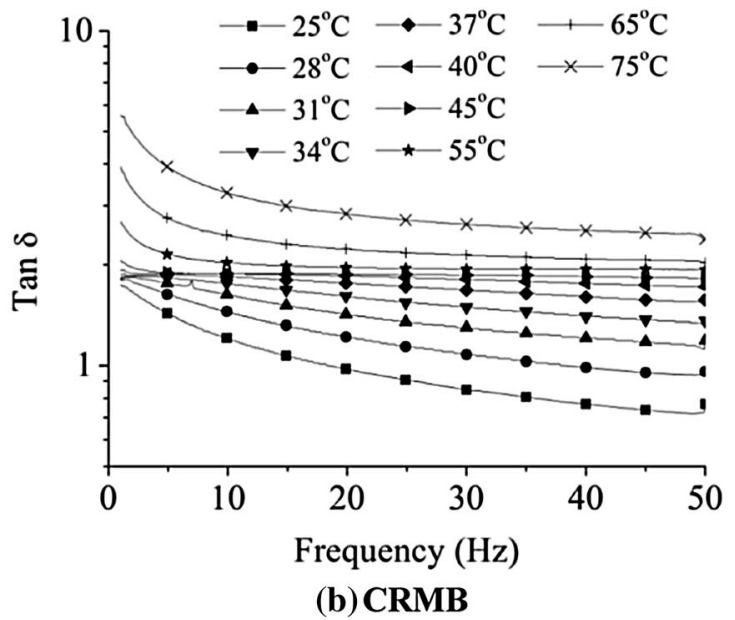

at $34{ }^{\circ} \mathrm{C}$ ). Hence the transition regime is considered from 34 to $37{ }^{\circ} \mathrm{C}$ for CRMB. The transition regime has shifted by approximately $6{ }^{\circ} \mathrm{C}$ upon short-term aging uniformly for both materials. Though both materials had different proportion of formation of oxidation products, it did not significantly influence the transition regime.

\section{Frequency Independent $\tan \delta$}

The frequency independence of $\tan \delta$ is shown in Fig. 6 . For unmodified bitumen, it can be seen that no frequency independent $\tan \delta$ is observed for temperatures varying from 22 to $65{ }^{\circ} \mathrm{C}$ (Fig. 6a). This shows that the behaviour of bitumen is different as that compared to polymers for which such criterion was discussed. The multi-constituent nature of bitumen responsible for a broad transition regime also could be a reason for such behaviour. However, the 
interesting fact is that for the crumb rubber modified bitumen, the frequency independent $\tan \delta$ is observed in the temperature range of $40-55^{\circ} \mathrm{C}$ (Fig. 6b).

A frequency independent $\tan \delta$ has been observed for bitumen modified with polyphosphoric acid while the base bitumen did not show such behaviour (Giavarini et al. [5]). No hypothesis or detailed explanation pertaining to the changes in microstructure causing such behaviour in modified bitumen is presented in this study.

The modifiers containing rubber such as PMB-E (elastomer modified bitumen) and CRMB absorb saturates in bitumen and swell in volume. Brule (1996) have shown that, an SBS concentration of $7 \%$ can swell to such an extent that the bitumen molecules are dispersed in the rubbery matrix. At this juncture, the transitions associated with the modifier also have to be considered. For instance, SBS shows two transitions, one at $-100{ }^{\circ} \mathrm{C}$ due to polybutadiene and another between 60 and $70{ }^{\circ} \mathrm{C}$ due to polystyrene blended with polybutadiene [11]. In this study, it was stated that the $T_{g}$ of both PS and PB are expected to further shift when blended with bitumen. The magnitude of such shift is yet to be quantified. The frequency independent $\tan \delta$ for PMB-E is observed in the range of $55-65{ }^{\circ} \mathrm{C}$. One can associate the transition occurring in this temperature range to that of PS in SBS. Though any literature scarcely exists explaining such behavior for CRMB, one can make

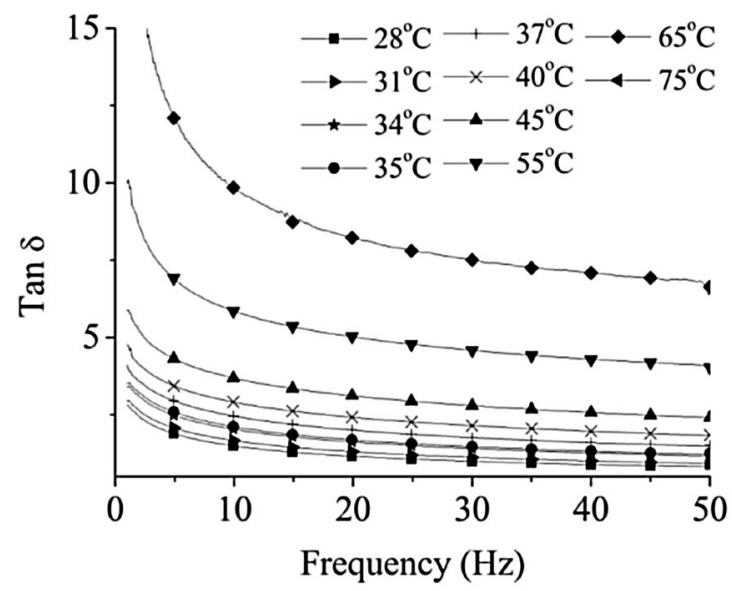

(a) VG30 similar arguments and associate the frequency independent $\tan \delta$ observed in CRMB to the rubber constituent in crumb rubber.

Upon short-term aging, the magnitude of $\tan \delta$ has reduced for short-term aged VG30 compared to its unaged condition (Fig. 7a). This shows the increased stiffness for short-term aged materials compared to the unaged condition. However, no such frequency independent $\tan \delta$ is observed in the short-term aged condition. For CRMB, the frequency independent region ranges from 55 to $65^{\circ} \mathrm{C}$ (Fig. 7b). An approximate $10{ }^{\circ} \mathrm{C}$ increase in transition regime is observed upon short-term aging for CRMB, the magnitude similar to that observed for $G^{\prime}-G^{\prime \prime}$ cross over. This parameter, however, can be used to estimate the transition temperature only for modified bitumen and not for unmodified materials.

The transition regime observed from $G^{\prime}$ to $G^{\prime \prime}$ cross over and frequency independence of $\tan \delta$ is summarized in Table 1. It can be seen that the transition temperature obtained from the $G^{\prime}-G^{\prime \prime}$ cross over is lower in magnitude compared to that observed from tan $\delta$ for CRMB. The transition temperature obtained from $\mathrm{G}^{\prime}$ to $\mathrm{G}^{\prime \prime}$ cross over corresponds to that of the modified bitumen rather than modifier in the presence of bitumen. Additional experiments have to be performed and more parameters identified to obtain a clear estimate of the transition temperature.

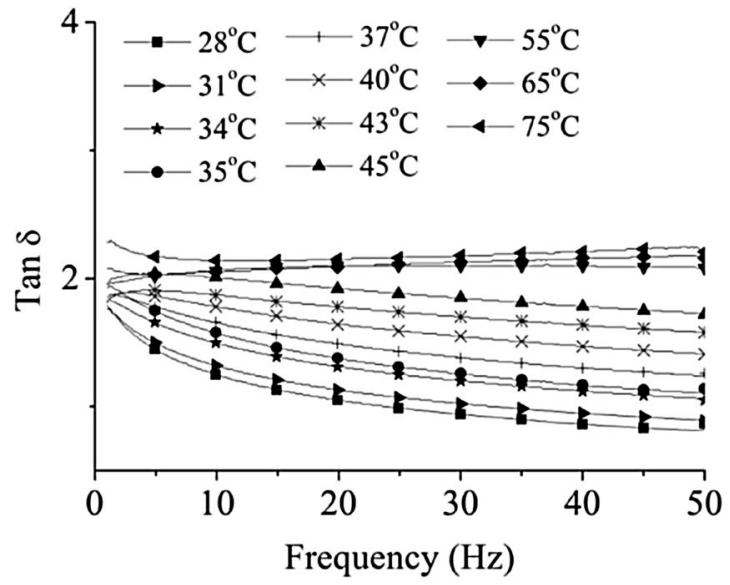

(b) CRMB

Fig. 7 Tan $\delta$ in short-term aged condition

Table 1 Transition temperature from frequency sweep test $\left({ }^{\circ} \mathrm{C}\right)$

\begin{tabular}{llllll}
\hline Material & \multicolumn{2}{l}{$\mathrm{G}^{\prime}-\mathrm{G}^{\prime \prime}$ cross over } & & \multicolumn{2}{l}{ Frequency independence of $\tan \delta$} \\
\cline { 2 - 3 } & Unaged & Short-term aged & & Unaged & Short-term aged \\
\hline VG30 & $25-28$ & $31-34$ & & - & - \\
CRMB & $28-31$ & $34-37$ & & $40-55$ & $55-65$ \\
\hline
\end{tabular}




\section{Conclusion}

Estimation of transition temperature for bitumen is highly complicated as it is difficult to identify parameters which can classify the solid-like/fluid-like behaviour of bitumen. Though tests in time domain and frequency domain can be performed on bitumen, the frequency domain tests are preferred. A frequency sweep test commonly performed to estimate the transition temperature for polymers was adopted in this study for bitumen. Two parameters, $G^{\prime}-G^{\prime \prime}$ cross over and frequency independence of $\tan \delta$ were used to estimate the transition temperature for bitumen. From the $G^{\prime}$ to $G^{\prime \prime}$ cross over, it was observed that the transition temperature of unmodified bitumen and crumb rubber modified bitumen varied from $25-28$ to $28-31{ }^{\circ} \mathrm{C}$ respectively. Upon short-term aging, a $6{ }^{\circ} \mathrm{C}$ increase in transition temperature was observed for VG30 and CRMB. It was also seen that the presence of the modifier reduced the cross over frequency in spite of a reduction in stiffness while short-term aging showed a reduction in cross over frequency for increase in stiffness. The cross over frequency and thus the transition regime were observed to be independent of the variation in stiffness.

The frequency independent $\tan \delta$ was not observed for the unmodified bitumen while it was remarkably clear for the crumb rubber modified bitumen. For the crumb rubber modified bitumen, the frequency independent $\tan \delta$ was observed from 40 to $55{ }^{\circ} \mathrm{C}$ in the unaged condition and from 55 to $65{ }^{\circ} \mathrm{C}$ in the short-term aged condition. The transition temperature obtained from frequency independent $\tan \delta$ corresponds to the modifier in the presence of bitumen. This parameter showed higher values of transition temperature compared to $G^{\prime}-G^{\prime \prime}$ cross over. However, the effect of aging was clearly seen from this parameter and was of similar magnitude when estimated from both parameters.

Acknowledgment This research work was supported by Department of Science and Technology, Govt. of India vide grant number: DST/TSG/STS/2011/46. This support is gratefully acknowledged.

\section{References}

1. Winter HH, Mours M (1997) Rheology of polymers near liquidsolid transitions. Adv Polym Sci 134:165-234

2. Padmarekha A, Krishnan JM (2013) Viscoelastic transition of unaged and aged asphalt. J Mater Civ Eng 25(12):1852-1863

3. Chambon F, Winter HH (1985) Stopping of crosslinking reaction in a PDMS polymer at the gel point. Polym Bull 13:499-503

4. Winter HH (1987) Can the gel point of a cross-linking polymer be detected by the $\mathrm{G}^{\prime}-\mathrm{G}^{\prime \prime}$ crossover? Polym Eng Sci 27(22):1698-1702

5. Giavarini C, Mastrofini D, Scarsella M (2000) Macrostructure and rheological properties of chemically modified residues and bitumens. Energy Fuels 14:495-502

6. Presti DL (2013) Recycled tyre rubber modified bitumens for road asphalt mixtures: a literature review. Constr Build Mater 49:863-881

7. Nivitha MR, Prasad E, Krishnan JM (2015) Ageing in modified bitumen using FTIR spectroscopy. Int J Pavement Eng. doi:10. 1080/10298436.2015.1007230

8. IS73:2013 (2013) Indian Standard for Paving Bitumen-Fourth Revision, Bureau of Indian Standards

9. IS15462:2004 (2004) Indian Standard for Polymer and Rubber Modified Bitumen, Bureau of Indian Standards

10. ASTM D2872 (2012) Standard test method for effect of heat and air on a moving film of asphalt (rolling thin-film oven test). ASTM International, West Conshohocken, PA

11. Masson JF, Bundalo-Perc S, Delgado A (2005) Glass transitions and mixed phases in block SBS. J Polym Sci Part B Polym Phys 43(3):276-279 